\title{
Cirugía retroperitoneal laparoscópica; experiencia de los 6 primeros meses del Hospital la Paz
}

\author{
A. Aguilera Bazán, S. Alonso y Gregorio, R. Cansino Alcaide, L. Hidalgo Togores, \\ J. Cisneros Ledo, J. de la Peña Barthel
}

Servicio de Urología. Hospital Universitario La Paz. Madrid.

Actas Urol Esp 2005; 29 (7): 657-661

\section{RESUMEN}

CIRUGÍA RETROPERITONEAL LAPAROSCÓPICA; EXPERIENCIA DE LOS 6 PRIMEROS MESES

DEL HOSPITAL LA PAZ

Presentamos los resultados iniciales del programa de cirugía laparoscópica retroperitoneal del Hospital Universitario La Paz desde julio de 2004 hasta diciembre de 2004. El programa se inició tras 2 años de experiencia en cirugía laparoscópica pélvica. La experiencia inicial ha sido lo suficientemente buena como para reducir progresivamente las contraindicaciones y aumentar el número de cirugias realizadas con dicha técnica.

Palabras clave: Curva de aprendizaje. Conversión. Complicaciones laparoscópicas. Nefrectomía laparoscópica.

\section{ABSTRACT}

RETROPERITONEAL LAPAROSCOPIC SURGERY; INITIAL EXPERIENCE IN THE HOSPITAL LA PAZ

We present the initial results of retroperitoneal laparoscopic surgery in the Hospital Universitario La Paz from july 2004 to december 2004. The program started after 2 years of pelvic laparoscopy surgery practice. The initial experience has been so good that we have reduced the contraindications for this technique and we have increased the number of laparoscopic surgery cases.

Keywords: Learning curve. Conversion. Laparoscopic complications. Laparoscopic nephrectomy.

L a cirugía laparoscópica retroperitoneal, aunque inicialmente puede parecer menos exigente que la cirugía pélvica (prostatectomía, cistectomía) presenta una serie de inconvenientes que debemos tener en cuenta a la hora de iniciar un programa de laparoscopia urológica. Uno de estos inconvenientes es la presencia de los grandes vasos en la zona donde vamos a trabajar, lo que aumenta el riesgo si carecemos de experiencia en dicha técnica.

En todo programa de cirugía laparoscópica urológica una de las primeras preguntas que nos planteamos es si comenzar con la cirugía retroperitoneal o por el contrario comenzar con la cirugía pélvica, principalmente prostatectomía ra- dical laparoscópica. Los defensores de la primera opción se basan, no sin cierta lógica, en que la cirugía retroperitoneal suele ser menos exigente desde el punto de vista técnico que la prostatectomía radical; por el contrario en aquellos hospitales en los que se comenzó con la cirugía pélvica, como es nuestro caso, la decisión se basó en el mayor número de casos disponibles para esta cirugía y por tanto la ventaja de conseguir una curva de aprendizaje menos prolongada.

En nuestro Servicio se decidió comenzar con el programa de cirugía laparoscópica retroperitoneal tras el desarrollo de la prostatectomía laparoscópica durante dos años. El cirujano encargado de ello ya había instaurado seis meses antes 
un programa de cirugía laparoscópica tanto retroperitoneal como pélvica en el Hospital de Getafe (Madrid), donde actualmente continúa realizándose este tipo de técnica.

En este artículo presentamos nuestra experiencia inicial en los seis primeros meses de laparoscopia retroperitoneal en el Servicio de Urología del Hospital La Paz.

\section{MATERIAL Y MÉTODOS}

El periodo de tiempo en estudio abarca desde julio de 2004 hasta diciembre de 2004, ambos inclusive. En un principio se desestimaron aquellos casos de tumores grandes (superiores a 7-10 cm) así como aquellos pacientes que hubieran sufrido cirugías renales previas. En los siete primeros casos se empleó el sistema de GelPort de Aesculap para realizar la nefrectomía; en los siguientes casos se dejó de emplear y se realizó la técnica pura. El número total de cirugías retroperitoneales realizadas fue de 19 nefrectomías (9 radicales, 7 simples, 3 nefroureterectomías) y 10 procedimientos varios ( 2 pieloplastias, 3 litiasis, 2 tumorectomias renales, 1 biopsia retroperitoneal, 1 suprarrenalectomía, 1 quiste renal). Todas las intervenciones se realizaron por vía transperitoneal excepto la decorticación del quiste que se realizó por retroperitoneoscopia (Tabla 1).

Tabla 1

\begin{tabular}{llc}
\hline \multicolumn{1}{c}{ No Casos } & & Técnica \\
\hline Nefrectomía radical & 9 & Transperitoneal \\
Nefrectomía simple & 7 & Transperitoneal \\
Nefroureterectomía & 3 & Transperitoneal \\
Tumorectomía & 2 & Transperitoneal \\
Suprarrenalectomía & 1 & Transperitoneal \\
Pieloplastia & 2 & Transperitoneal \\
Litiasis & 3 & Transperitoneal \\
Quiste renal & 1 & Lumboscopia \\
Biopsia retrop. & 1 & Transperitoneal \\
\hline
\end{tabular}

La técnica quirúrgica empleada principalmente fue el abordaje transperitoneal con el paciente en decúbito lateral y la generación del neumope- ritoneo una vez colocado el puerto de mano o en los casos de laparoscopia pura, una minilaparotomía de $2 \mathrm{~cm}$ aproximadamente que luego emplearíamos para ubicar la óptica. Se trabajó con 4 puertos ( 2 de $12 \mathrm{~mm}, 2$ de $5 \mathrm{~mm}$ ) y óptica de $30^{\circ}$ (Wolf). El clipaje de la arteria renal se realizó con Hem-o-lok de 10 mm (Wolf) y la vena con EndoGia de 30/2,5 mm (Autosuture). La extracción de la pieza se realizó por el puerto de mano o por una incisión de McBurney en el caso de laparoscopia pura.

\section{RESULTADOS}

La serie de nefrectomías estaba formada por 10 varones y 9 mujeres con una media de edad de 52,26 años (22-79), 10 de ellos eran ASA 1, 5 ASA 2 y 4 ASA 3. En 6 casos existían antecedentes de cirugías abdominales previas (apendicectomía, herniorrafia inguinal, histerectomía con doble anexectomía, cirugía de coraliforme). 8 nefrectomías fueron derechas y 11 izquierdas. El tiempo quirúrgico medio empleado fue de 190 minutos (150-270), siendo las nefroureterectomías las que requirieron mayor tiempo operatorio. El sangrado medio operatorio fue de $158,82 \mathrm{ml}$ (50-500), requiriendo transfusión 2 casos, uno de ellos preoperatoria debido al estado de anemización en que se encontraba la paciente (paciente $\mathrm{n}^{\circ}$ 17, nefroureterectomía). El tamaño medio de la incisión para extraer la pieza fue de $7,2 \mathrm{~cm}$ (4-12), teniendo en cuenta que en los primeros casos se empleaba el puerto de mano $(7,5-8 \mathrm{~cm})$ situado en la línea media supra o infraumbilical según el caso. En el apartado de complicaciones intraoperatorias hubo una lesión vascular que requirió reconversión a cirugía abierta (paciente $\mathrm{n}^{\mathrm{o}}$ 1) y una lesión de vena lumbar que se logró controlar por laparoscopia (paciente $\mathrm{n}^{\circ}$ 10, 500 cc de sangrado). La estancia media hospitalaria fue de 5,4 días (3-30), siendo el caso $\mathrm{n}^{\mathrm{o}} 1$ el de mayor estancia por lesión vascular. Con la excepción de este primer caso, la estancia media quedaría en 4 días de ingreso postoperatorio. Entre las complicaciones postoperatorias se produjo 1 edema agudo de pulmón (paciente $\mathrm{n}^{\circ}$ 14, 9 días de ingreso), 1 angina de pecho en paciente con antecedentes de IAM (paciente $\mathrm{n}^{\circ}$ 15, 5 días de ingreso) y 1 sangrado postoperatorio (paciente $\mathrm{n}^{\circ} 5,7$ días 
de ingreso) que requirió revisión quirúrgica, evidenciándose un sangrado de una rama perforante del psoas. En cuanto al análisis de las piezas, 7 fueron atrofias postobstructivas, 7 pT1NOMO, 5 pT3G3NOMO.

Se produjo una muerte intraoperatoria en el paciente $\mathrm{n}^{\mathrm{o}}$ 9, varón de 53 años, con sobrepeso (110 kg, IMC 33,6), HTA, hipertrofia de ventrículo izquierdo, hipercolesterolemia, aneurisma abdominal, fumador, riesgo anestésico ASA 4, diagnosticado de un tumor renal, al que se le realizó una nefrectomía radical izquierda en 2 horas con escaso sangrado (aproximadamente de $150 \mathrm{cc}$ ), que presentó una taquicardia supraventricular intraoperaroria que le provocó un paro cardíaco sin respuesta a maniobras de reanimación cardio-pulmonar. La familia no autorizó la necropsia, no pudiéndose determinar la causa de la muerte.

La serie de otros procedimientos retroperitoneales constaba de 10 pacientes, 6 varones y 4 mujeres con una media de edad de 53,8 años (30-74). 5 de estos pacientes eran ASA 1, 2 ASA 2 y 3 ASA 3. 4 casos presentaban antecedentes de cirugias abdominales previas (apendicectomía, herniorrafia inguinal, quiste hidatídico hepático, cirugía reconstructiva de eventración). El tiempo medio operatorio fue de $140 \mathrm{~min}$ (90-210, este último en el paciente $\mathrm{n}^{\circ} 7$ al que se le practicó una tumorectomía renal de $4 \mathrm{~cm}$ ). El sangrado operatorio fue insignificante salvo en el paciente de la tumorectomía de $4 \mathrm{~cm}$ de diámetro que tuvo un sangrado de $500 \mathrm{ml}$, sin llegar a precisar transfusión. Las incisiones realizadas fueron de escasa importancia ya que las piezas extirpadas eran de tamaño pequeño (litiasis, tumorectomías, suprarrenal, adenopatías), pudiendo extraerse por el trócar de $12 \mathrm{~mm}$ ampliado 1-2 cm. No hubo complicaciones intraoperatorias importantes, no fue necesaria la reconversión en ninguno de los casos. La estancia media hospitalaria fue de 3,2 días (2-7, este último caso fue en la paciente $n^{\circ} 3$ operada de pieloplastia que sufrió una gastroenteritis aguda en el postoperatorio inmediato). Entre las complicaciones postoperatorias cabe destacar 1 revisión quirúrgica en el paciente $n^{\circ} 2$ por

Tabla 2 salida de una punta de epiplon por el trayecto de un trócar de $10 \mathrm{~mm}$ lo que prolongó su postoperatorio a 4 días (Tabla 2).

En este mismo periodo de tiempo se realizaron también 29 procedimientos retroperitoneales por cirugia abierta, 18 varones y 11 mujeres con una edad media de 55 años, de los cuales 21 fueron nefrectomías (8 radicales, 9 simples, 4 donante vivo) y 8 cirugias variadas (3 litiasis renales, 1 suprarrenal, 1 fibrosis retroperitoneal, 2 nefrectomías parciales, 1 estenosis de la unión pieloureteral). La estancia media postoperatoria de las nefrectomías fue de 5,8 días (3-15 días) y el de las cirugias variadas de 6,5 días (3-13). Entre las complicaciones postoperatorias nos encontramos con 3 casos que requirieron transfusión, 1 caso de angor vasoespástico y otro de derrame pleural que requirió drenaje.

\section{CONCLUSIONES Y DISCUSIÓN}

Como se ha comentado en la introducción, el caso de nuestro Hospital no es un caso típico de inicio de un programa de laparoscopia, ya que sin experiencia previa en dicha técnica se decidió comenzar por la cirugía más compleja debido al gran número de casos que se presentaban al año, con el fin de reducir al máximo la curva de aprendizaje que entraña la laparoscopia ${ }^{1}$. Una vez adquirida experiencia e instaurada una sistemática de trabajo se decidió comenzar a realizar cirugia retroperitoneal. Si bien aparentemente es más sencilla, hay un detalle importante que creo nos pone a todos los urólogos en alerta cuando vamos a comenzar este tipo de cirugía: un accidente vascular en laparoscopia es muy difícil resolver mediante dicha técnica y en el campo del que estamos hablando la presencia de la aorta y la cava con sus múltiples ramas y su variabilidad anatómica hace que esta posibilidad no sea descabellada $^{2}$. A esta situación debemos añadir la desorientación que suele acompañar al hecho de trabajar con una óptica a escasos centímetros del

\begin{tabular}{lccccc}
\hline & Edad & Tiempo & Sangrado & Transfusión & Estancia \\
\hline Nefrectomias & 52 & $190 \mathrm{~min}$ & $158,82 \mathrm{cc}$ & $2^{*}$ & 4 días \\
Otras & 53 & $140 \mathrm{~min}$ & $55 \mathrm{cc}$ & 0 & 3,2 dias \\
\hline
\end{tabular}

*Un caso de transfusión preoperatoria 
lugar de disección, así como la clara dependencia del ayudante en el manejo de la cámara, sobre todo si estamos trabajando con una óptica de $30^{\circ}$ con la cual la pérdida de referencias suele ser más habitual.

Teniendo en cuenta todos estos factores, se decidió que en las primeras intervenciones de este nuevo programa empleáramos el puerto de mano en las nefrectomías simples o radicales e hiciéramos dichas cirugias asistidas por la mano. Tras revisar el mercado y probar diversos modelos, Omniport de Autosuture, LapDisc de Ethicon, GelPort de Aesculap, nos decantamos por este último por presentar algunas ventajas sobre sus competidores (introducción de instrumental a través de la membrana sin pérdida de neumoperitoneo, mayor facilidad para la introducción-extracción de la mano) $)^{3,4}$. La dificultad del montaje de dicho puerto es muy similar al de los otros puertos de mano y su principal desventaja radica en el precio (aproximadamente $600 €$ por unidad). El manejo de los puertos de mano en general nos aporta varias ventajas respecto a la laparoscopia pura; tener una mano dentro del abdomen nos da más confianza ante la posibilidad de sufrir un accidente vascular; tener una referencia visual en nuestra mano hace que el cerebro asimile con más facilidad la pérdida de la tercera dimensión y por tanto acorta la curva de aprendizaje; por último, no perder la sensación del tacto permite realizar determinadas maniobras como es la identificación de estructuras vasculares. A pesar de todo ello, aunque inicialmente el manejo de la mano sólo nos aporta ventajas, a medida que se va adquiriendo experiencia en esta cirugía y este campo anatómico, nos damos cuenta que el tener una mano prácticamente hipotecada como separador nos resta eficacia a la hora de la disección más fina de estructuras y que nuestra progresión puede verse afectada. Aunque se trata de una percepción personal, nos da la impresión de que el postoperatorio en estos pacientes es algo más tórpido en lo que se refiere a ileo intestinal. Finalmente, tras adquirir confianza y cierta experiencia en este tipo de técnica decidimos realizar las cirugias sin ayudarnos ni colocar el dispositivo para la mano. Esta decisión hace que seamos todavía más prudentes a la hora de manejar el hilio renal o las diversas ramas que proceden de los grandes vasos; un sangrado importante puede poner en peligro la vida del paciente, pero un sangrado menos grave puede dificultar mucho la posterior disección de los planos anatómicos o el reconocimiento de estructuras.

En lo que se refiere a la selección de los casos, inicialmente evitamos realizar nefrectomías en tumores grandes $(>7 \mathrm{~cm}$ ) que pudieran dificultar el manejo de la pieza. También procuramos evitar aquellos pacientes que ya habían sufrido una cirugía sobre el riñón que se iba a quitar o pacientes que presenten radioterapia o cirugías abdominales previas sobre el área próxima a la celda renal. Sin embargo poco a poco estas contraindicaciones se fueron modificando y se realizaron cirugias sobre tumores grandes o nos planteamos el abordaje retroperitoneoscópico en casos en que sospechemos un abordaje transperitoneal dificultoso, como fue el caso del paciente $\mathrm{n}^{\circ} 10$ con antecedentes de dos cirugias de quiste hepático hidatídico y colocación de dos mallas por eventración que presentaba un quiste renal gigante de $25 \times 20$ $\mathrm{cm}$ sintomático. Posteriormente hemos realizado más lumboscopias (suprarrenalectomías, biopsias retroperitoneales) y aunque no creemos que sea la principal vía de abordaje, si que debe ser una técnica que debemos conocer y saber realizar ya que puede haber casos en que la técnica transperitoneal no sea posible o sea muy dificultosa ${ }^{5}$. Sin duda el espacio de trabajo en el caso de la lumboscopia es mucho más reducido con lo que eso implica a la hora de la colocación de trócares y dificulta la cirugía reconstructiva como puede ser la pieloplastia; además, creemos que la facilidad con la que podemos desorientarnos es mayor y si el paciente es obeso la dificultad aumenta de manera importante.

En lo que se refiere a la técnica empleada, como se ha indicado anteriomente, se empleó el abordaje transperitoneal ya que el espacio distendido es mayor facilitando así la colocación de trócares y mejorando la orientación respecto al abordaje retroperitoneoscópico. Se intentó triangular la colocación de trócares, de manera que la óptica se encuentre entre las dos manos del cirujano. En este sentido, el triángulo formado por los tres puertos se desplazó lateral o medialmente según el fenotipo del paciente y superior o inferiormente según la zona sobre la que íbamos a operar. 
Como conclusiones queremos destacar la experiencia de nuestro Servicio comenzando por la prostatectomía radical como inicio del programa laparoscópico no es una mala opción, aunque habría que personalizar en cada caso ya que puede haber otros servicios que por menor volumen o por cualquier otro factor limitante tengan muy difícil comenzar por esta técnica. En estos casos no nos parece una mala práctica iniciarse en la cirugía retroperitoneal pero con mucha prudencia y seleccionando bien los casos, ya que un accidente en este campo quirúrgico suele traer consecuencias funestas y puede ser el final del programa de laparoscopia. Por otra parte, el empleo de puertos de mano para ayudarnos en las nefrectomías creemos que es una buena práctica inicial para tomar confianza y soltura, pero se debe abandonar en cuanto la curva de aprendizaje sea la adecuada.

En cuanto a la comparación entre cirugía laparoscópica y cirugía abierta, son múltiples los estudios que han demostrado que la laparoscopia es un tratamiento técnicamente correcto, con menor morbilidad y con un control de la enfermedad a medio plazo equivalente al de la cirugía abierta. Como desventaja, suele precisar de un mayor tiempo operatorio y generalmente un mayor coste inicial en la implantación de la técnica $^{6}$. Nuestra serie, todavía reducida y de corto seguimiento parece seguir esta línea, apoyando la idea de que la cirugía retroperitoneal laparoscópica terminará siendo el gold standard en la mayoría de los casos.

\section{REFERENCIAS}

1. Fahlenkamp, D., Rassweiler, J., Fornara, P., Frede, T. and Loening, S. A.: Complications of laparoscopic procedures in urology: experience with 2,407 procedures at 4 German centers. J Urol 1999 Sep;162(3 Pt 1):765-770.

2. Parsons JK, Varkarakis I, Rha KH, Jarrett TW, Pinto PA, Kavoussi LR. Complications of abdominal urologic laparoscopy: longitudinal five-year analysis. Urology 2004 Jan; 63(1):27-32.

3. Pietrow, Paul K, Albala, DM. Hand-assisted urological laparoscopy. Curr Opin Urol 2002 May; 12(3):233-7.

4. Lotan Y, Duchene DA, Cadeddu JA, Koeneman KS. Cost Comparison of Hand Assisted Laparoscopic Nephrectomy and Open Nephrectomy: Analysis of Individual Parameters. J Urol 2003 Sep;170(3):752-5.

5. Guillonneau B. Ballanger P. Lugagne PM. Valla JS. Vallancien G. Laparoscopic versus lumboscopic nephrectomy. [Clinical Trial. Controlled Clinical Trial. Journal Article. Multicenter Study] Eur Urol 29(3):288-91, 1996

6. Meaney A, Anoop M., Gill Inderbir S. Financial analysis of open versus laparoscopic radical nephrectomy and nephroureterectomy. J Urol 2002 Apr;167(4):1757-62.

Dr. A. Aguilera Bazán

Po de la Castellana 261. 28046 Madrid

Servicio de Urología, Hospital La Paz.

Email: aaguilera11@yahoo.es

(Trabajo recibido el 30 marzo de 2005)

\section{COMENTARIO EDITORIAL}

Los autores presentan su experiencia en cirugía retroperitoneal tras una importante actividad en prostatectomía radical laparoscópica. En el artículo exponen el uso de la cirugía asistida por la mano para posteriormente pasar a laparoscopia pura.

En mi opinión, es muy importante la llamada a la prudencia que hacen los autores basada en la importante morbilidad que esta cirugía puede llegar a tener. Este es un hecho que todos los grupos que hemos incorporado la laparoscopia he- mos experimentado, creo que sin excepción, y por ello hemos de estar preparados para ello, a la vez que creo oportuno informar a nuestros pacientes de esta circunstancia. Por esto, creo esencial que la incorporación de la laparoscopia debe ser una decisión estratégica de un Servicio y que debe contar con el respaldo de todo el grupo. Sólo así se puede avanzar y alcanzar los excelentes resultados que los autores presentan. 